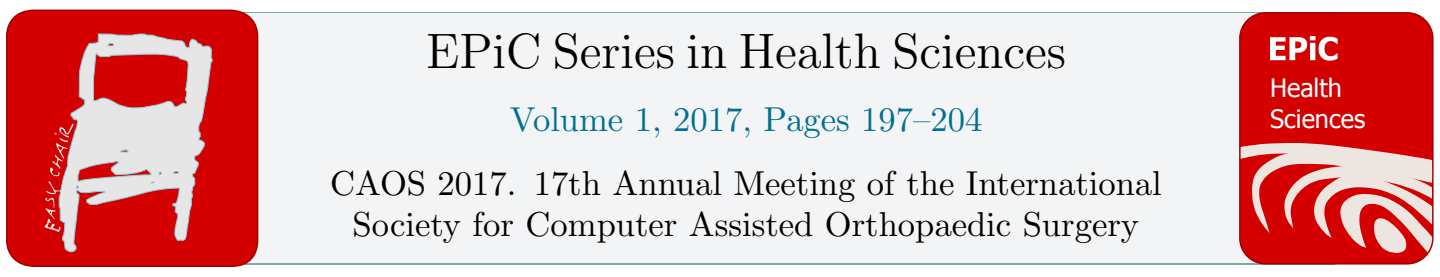

\title{
A Novel Method for Defining Ligament Characteristics in Subject Specific Dynamic Surgical Planning
}

\author{
Willy Theodore BE (Hons) $)^{1,5 *}$, Joseph Little BE ${ }^{1}$, David Liu MD ${ }^{2}$, Jonathan \\ Bare $\mathrm{MD}^{3}$, David Dickison $\mathrm{MD}^{4}$, Professor Mark Taylor $\mathrm{PhD}^{5}$, Brad Miles \\ $\mathrm{PhD}^{1}$ \\ ${ }^{1} 360$ Knee Systems, Sydney, 2073, Australia \\ ${ }^{2}$ Bone and Joint Surgery Gold Coast, Gold Coast, 4224, Australia \\ ${ }^{3}$ Melbourne Orthopaedic Group, Melbourne, 3181, Australia \\ ${ }^{4}$ Penninsula Orthopaedics, Sydney, 2099, Australia \\ ${ }^{5}$ Flinders University, Adelaide, 5001, Australia \\ willy@kneesystems.com
}

\begin{abstract}
Despite of the high success of TKA, 20\% of recipients remain dissatisfied with their surgery. There is an increasing discordance in the literature on what is an optimal goal for component alignment. Furthermore, the unique patient specific anatomical characteristics will also play a role. The dynamic characteristics of a TKR is a product of the complex interaction between a patient's individual anatomical characteristics and the specific alignment of the components in that patient knee joint. These interactions can be better understood with computational models. Our objective was to characterise ligament characteristics by measuring knee joint laxity with functional radiograph and with the aid of a computational model and an optimisation study to estimate the subject specific free length of the ligaments.

Pre-operative CT and functional radiographs, varus and valgus stressed X-rays assessing the collateral ligaments, were captured for 10 patients. CT scan was segmented and 3D-2D pose estimation was performed against the radiographs. Patient specific tibio-femoral joint computational model was created. The model was virtually positioned to the functional radiograph positions to simulate the boundary conditions when the knee is stressed. The model was simulated to achieve static equilibrium. Optimisation was done on ligament free length and a scaling coefficient, flexion factor, to consider the ligaments wrapping behaviour.

Our findings show the generic values for reference strain differ significantly from reference strains calculated from the optimised ligament parameters, up to $35 \%$ as percentage strain. There was also a wide variation in the reference strain values between
\end{abstract}


subjects and ligaments, with a range of $37 \%$ strain between subjects. Additionally, the knee laxity recorded clinically shows a large variation between patients and it appears to be divorced from coronal alignment measured in CT. This suggest the ligaments characteristics vary widely between subjects and non-functional imaging is insufficient to determine its characteristics. These large variations necessitates a subject-specific approach when creating knee computational models and functional radiographs may be a viable method to characterise patient specific ligaments.

\section{Introduction}

Total Knee Arthroplasty (TKA) is a highly successful surgical intervention that relieves pain and improves quality of life for osteoarthritis sufferers. Despite this, as many as $20 \%$ of recipients remain dissatisfied with their surgery (Bourne et al., 2010). Major reasons for post-operative dissatisfaction include recurrent pain and functional impairment post-surgery. (Beswick et al., 2012; Baker et al., 2007).

Component alignment has been shown to influence outcomes (Huang et al., 2012), with relationships between femoral and tibial component axial plane orientation being some of the most compelling evidence to support this (Nicoll et al., 2010; Bell et al., 2014, Barrack et al., 2001). However, there is an increasing discordance in the literature on what is an optimal goal for component alignment. More traditional philosophies that standardise component alignment to biomechanical axis are being challenged by subject specific alignment philosophies. With proponents of all techniques showing satisfactory and even improved outcomes. (Howell et al., 2014; Dossett et al., 2014). Component alignment has been shown to alter the dynamics of the knee (Harman et al., 2012; Carr et al., 2009; Werner et al., 2005). Furthermore, the unique patient specific anatomical characteristics will also play a role (Bellemans et al., 2002; Hutter et al., 2013). The final dynamic characteristics of a TKR will undoubtedly be a product of the complex interaction between a patient's individual anatomical characteristics and the specific alignment of the components in that patient knee joint.

These complex interactions may be better understood by looking at individual patient's dynamic characteristics with sophisticated computational models. Recent studies have widened the availability of musculoskeletal models to surgical planning for Total Joint Arthroplasty (Marra et al., 2015; Chen et al., 2014). These developments may help answer the beforementioned questions about what is an optimal goal for surgery by taking computational models into a computer assisted surgical planning environment.

Development of knee computational model is a continual challenge. Often, the details in published models varied considerably, including the number and complexity of included structures, material behaviour, or the use of generic versus subject-specific representations of bone and cartilage geometry (Harris et al., 2016). Regardless of the complexity, subject specific information has been identified as important inputs to knee models (Roth et al., 2015; Harris et al., 2016). Most TKR computational models use either CT or MRI as input. From this non-functional imaging, subject specific bony morphology and anatomical landmarks can be obtained but not the ligament characteristics. There have been few studies that able to estimate ligament soft tissue properties of cadavers from the knee laxity data recorded experimentally (Ewing et al., 2016; Baldwin et al., 2012; Bloemeker et al., 2012). However, the data and method used to collect the knee laxity in these experiments can be difficult to attain in a clinical setting because of cost and time implication. 
Therefore, we realise there is a gap in collecting the subject specific information needed to utilise computational models in a computer assisted surgical planning environment, particularly ligament characteristics. Our objective was to create a novel method to define the ligament characteristics by measuring knee joint laxity with a TELOS stress testing device and with the aid of a computational model and an optimisation study to estimate the subject specific free length of the ligaments. In the current study, we compared numerically obtained reference strains by optimising ligament parameters and the generic reference strains reported in literature.

\section{Method}

Clinical assessment of knee laxity: Pre-operative CT and functional radiographs, stressed X-rays with stress device (TELOS) were captured for 10 patients. The knee was stressed at 2 flexion angles, $20^{\circ}$ flexion and $90^{\circ}$ flexion. At each flexion angle, the knee is stressed for varus and valgus load. These stressed positions were selected to assess Lateral Collateral Ligament (LC) and Medial Collateral ligament (MCL). Radiographs were taken whilst the knee was held in the stressed position. The load was incrementally increased to either a maximum load of $150 \mathrm{~N}$ or until patient felt uncomfortable.

Image processing: CT scans were segmented using ScanIP software (Simpleware) and bony anatomical points, including ligament attachment sites were landmarked. 3D-2D pose estimations were performed in MIMICS - X-ray module (Materialise) using the segmented femur and tibia against the radiographs to determine knee alignment for each functional radiograph. Each registered femur and tibia is normalised to the $\mathrm{CT}$ reference frame. The 3D-2D registered femur is then registered to the femur in CT reference frame. Then transformation matrix of the tibia in CT reference frame to each of the 3D-2D registered tibia is calculated. The transformation matrix is used to transform the anatomical landmarks taken in the CT reference frame to the functional positions to determine the knee alignment for each functional radiograph. Femur and tibia mechanical axis were determined from anatomical landmarks and were used to calculate the knee alignment.

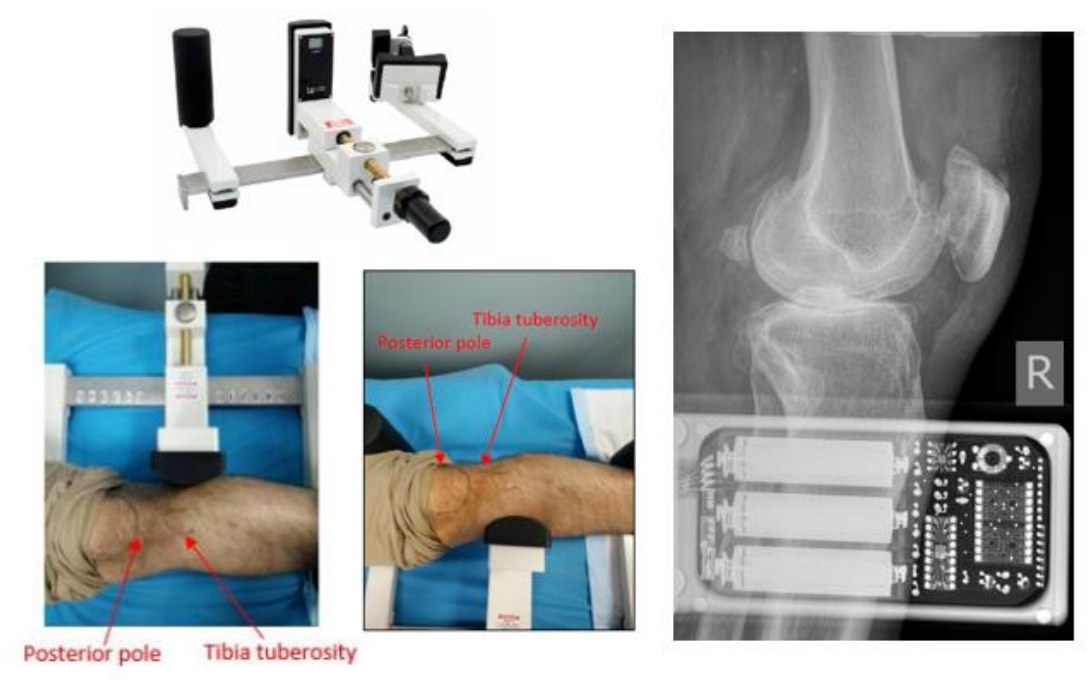

Figure 1. TELOS device and stressed X-rays for varus and valgus load. 
Tibio-femoral knee laxity model: Patient specific rigid body models were created in multibody dynamics simulation software, ADAMS (MSc) for native tibio-femoral joint. The model includes patient reconstructed bony geometries, anatomical landmarks and ligaments (LCL and MCL). The LCL was considered to be a single fibre bundle and the MCL was considered to consist of anterior and posterior bundles. Each ligament was modelled as 1D non-linear spring elements, shown in Equation (1):

$$
\begin{aligned}
& f=\frac{1}{4} k \frac{\varepsilon^{2}}{\varepsilon_{l}}, \quad 0 \leq \varepsilon \leq 2 \varepsilon_{l} \\
& f=k\left(\varepsilon-\varepsilon_{l}\right), \quad \varepsilon>2 \varepsilon_{l} \quad \text { Equation (1) } \\
& f=0, \quad \varepsilon<0 \\
& \varepsilon=\frac{l-l_{\text {free }}}{l_{\text {free }}}+(\text { flexion } * f f)
\end{aligned}
$$

Where $k$ is the stiffness parameter obtained from the literature (Li et al., 999), lfree is the free length of the ligament, and $\varepsilon_{l}$ is the spring parameter assumed to be 0.03 (Wismans et al., 1980). The free length is defined as the length of the ligament when it first becomes taut. Reference strain was defined as the strain in the ligament when the leg is in a neutral position, which in this study is defined with the leg in full extension in CT scan. Thus, reference length is the ligament length when the leg is in full extension in CT scan. The reference strain can be calculated then with Equation (2). The ligament length is calculated as 3D distance between attachment points and the strain is calculated as a function of flexion angle. The scaling coefficient, flexion factor $(f f)$, is introduced to take into account the wrapping of ligaments.

All components were modelled as rigid bodies using a penalty based contact between components. The model was virtually positioned to the functional radiograph positions to simulate the boundary conditions when the knee is stressed. TELOS load was applied and the model was simulated to achieve static equilibrium. Static equilibrium is reached when the moments caused by the external load, TELOS load, is neutralised by the internal load of the knee joint, contact forces and ligament forces. The model was allowed to translate superior-inferiorly while maintaining the knee alignment during simulation to induce contact between distal femur and proximal tibia.

Optimisation of ligament parameters: lfree and $f f$ were optimised to minimise differences between the resultant moment on the knee caused by the TELOS load and the simulated resultant moment with derived ligament parameters. Parameter optimisation was performed in Python using Newton Raphson method. The initial values of lfree were determined using the reference strains (expressed as percent strain) taken from literature (Wismans et al., 1980). The values are shown in Table 1. Subject specific reference strains were then calculated using Equation (2) with the optimised ligament parameters. 
(a)

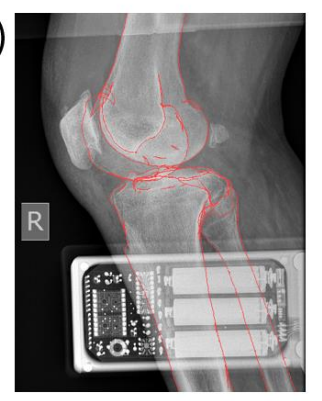

(b)

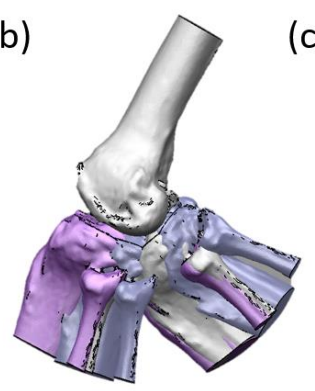

(c)

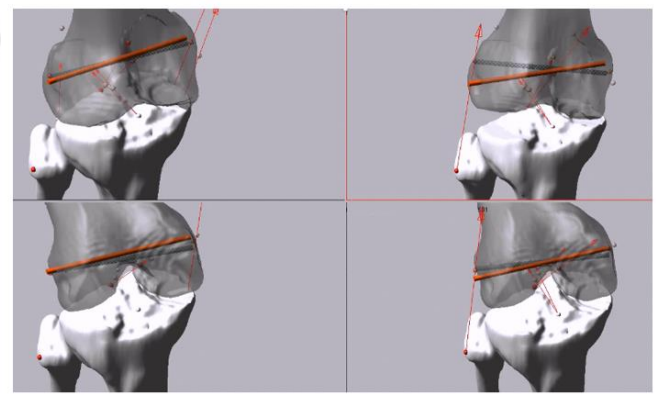

Figure 2. (a) 3D-2D registration of segmented femur and tibia. (b) the 3D-2D registered femur and tibia normalised to the $\mathrm{CT}$ reference frame; Transformation matrix of the tibia in $\mathrm{CT}$ reference frame to each of the registered position is calculated. (c) Tibio-femoral joint model replicates the knee position in functional radiographs.

\section{Results}

The mean coronal alignment in $\mathrm{CT}$ was $5^{\circ}$ varus, ranging from $8^{\circ}$ valgus to $12^{\circ}$ varus. Each subject had a varus valgus laxity calculated from the functional radiographs taken. The mean varus valgus laxity range in extension and flexion were $5^{\circ}\left(\mathrm{SD}, 3^{\circ}\right)$ and $3^{\circ}\left(\mathrm{SD}, 2^{\circ}\right)$ respectively. Subject specific reference strains determined from optimised ligament parameters are summarised in Table 1. There was a wide variation in the reference strain values between subjects and ligaments. The antMCL ligament showed the greatest variation in reference strain, with a range of $37 \%$ strain between subjects.

\section{Discussion}

Knee extension is usually considered as the reference state from which different motions can be simulated. In this state, the ligaments are strained and therefore already sustaining a load (Galbusera et al., 2014). Our findings show the generic values for reference strain differ significantly from numerically obtained reference strain. Furthermore, there was a wide variations in reference strains between subjects and ligaments, with variations ranging from $9 \%$ to $37 \%$ strain.

These reference strains affects the ligament free length used to calculate the ligament strain throughout the motion. The strains measured will affect the ligament force as shown in Equation (1) and it can potentially affect the outputs of a knee computational model. These large variations necessitates a subject-specific approach when creating knee computational models. Additionally, the knee joint laxity recorded clinically shows a large variation between patients and it appears to be divorced from coronal alignment measured in CT. This suggest the ligaments characteristics vary widely between subjects and non-functional imaging is insufficient to determine its characteristics.

Computational model is an emerging technology for TKA, especially its use in surgical planning environment. Subject specific information is needed in these models to better represent patient variability in the population (Roth et al., 2015; Harris et al., 2016). Few computational model studies (Ewing et al., 2016; Baldwin et al., 2012; Bloemeker et al., 2012) have been able to estimate ligament 
soft tissue properties of cadavers from the knee laxity data recorded experimentally. However, the data and method used to collect the knee laxity in these experiments can be difficult to attain in a clinical setting because of cost and time implication. This study demonstrates the development of a novel method to characterise subject specific ligament properties utilising functional radiographs and computational model.

\begin{tabular}{|c|c|c|c|c|c|c|}
\hline & \multirow{2}{*}{$\begin{array}{l}\text { LCL } \\
(\%)\end{array}$} & \multirow{2}{*}{$\begin{array}{c}\operatorname{antMCL} \\
(\%)\end{array}$} & \multirow{2}{*}{$\begin{array}{c}\text { postMCL } \\
(\%)\end{array}$} & \multirow{2}{*}{$\begin{array}{c}\text { Coronal } \\
\text { alignment } \\
\text { in CT }\left(^{\circ}\right)\end{array}$} & \multicolumn{2}{|c|}{$\begin{array}{c}\text { Varus valgus laxity } \\
\text { range }\end{array}$} \\
\hline & & & & & $\begin{array}{c}\text { extension } \\
\left({ }^{\circ}\right)\end{array}$ & $\begin{array}{c}\text { flexion } \\
\left({ }^{\circ}\right)\end{array}$ \\
\hline $\begin{array}{l}\text { Literature } \\
\text { value (Wismans } \\
\text { et al., 1980) }\end{array}$ & 5 & -3 & 5 & & & \\
\hline Subject 1 & 6 & 4 & 3 & 12 & 3 & 5 \\
\hline Subject 2 & 10 & 32 & 2 & 4 & 5 & 1 \\
\hline Subject 3 & 8 & 8 & -4 & 9 & 1 & 1 \\
\hline Subject 4 & 9 & 10 & 0 & 9 & 2 & 2 \\
\hline Subject 5 & 1 & 2 & 2 & 4 & 5 & 2 \\
\hline Subject 6 & 6 & 3 & 1 & 5 & 1 & 1 \\
\hline Subject 7 & 4 & -5 & -4 & 5 & 7 & 4 \\
\hline Subject 8 & -2 & -1 & -1 & 7 & 9 & 3 \\
\hline Subject 9 & 12 & 6 & 5 & 0 & 6 & 4 \\
\hline Subject 10 & 14 & 6 & -1 & -8 & 6 & 8 \\
\hline Average \pm std & $7 \pm 5$ & $7 \pm 10$ & $\mathbf{0} \pm 3$ & $5 \pm 6$ & $5 \pm 3$ & $3 \pm 2$ \\
\hline
\end{tabular}

Table 1. Optimised reference strains (as \% strain); Coronal alignment in CT scan and varus valgus laxity in extension and flexion (Varus angle is positive).

There are some limitations in this study. Firstly, the 3D-2D registration was done on single planar Xray. The 3D position of the knee is not fully captured with single planar X-ray, particularly the inplane rotation and depth of the knee. Bi-planar X-rays can provide 3D position of the knee however its use has not been standardised in imaging centres. Further verification of the single 3D-2D single planar X-ray registration is needed to minimise alignment calculation error. Secondly, no meniscus is modelled in the computational model. This may affect the contact force calculated in the simulation as one of meniscus's functions is to distribute the loads experienced by the knee. The current model is developed with CT scan as inputs and meniscus is not visible in CT. Part of future work is to adapt the model for magnetic resonance imaging (MRI) and include meniscus in the model. Thirdly, the optimisation was performed only on 2 parameters. Ligaments of the knee are among the most complicated structures to simulate. Most computational models simplified the ligament attachment areas as a single point to minimise computing cost and therefore limits the effective line of action movement of the ligament force in the model. Computing cost is a critical factor for a computational model to be used as surgical planning tool and thus the justification of the simplification. In this study, a variable was introduced to consider the wrapping behaviour of the ligament and it is varied based on flexion angle. Further work is to improve the mechanical role of the 1D non-linear spring by implementing the ability to simulate the movement of the force line of action, particularly in the 
anterior-posterior direction. With further development, this movement could be characterize with 1-2 additional parameters and solved for in a similar manner as for free length and flexion factor.

Patient specific computational modelling has the potential to help understand the complex interactions between a patient's individual anatomical characteristics and the specific alignment of the components in that patients knee joint. Reference strains calculated in our study are patient specific and incorporating them into patient specific computational models may help to account for this variability in the population. Utilisation of this technique in a computer assisted surgical planning environment is the next step. Our study demonstrated functional radiographs may be a viable method to characterise patient specific ligaments.

\section{References}

Baldwin, M.A., et al., Dynamic finite element knee simulation for evaluation of knee replacement mechanics, Journal of Biomechanics, vol. 45, no. 3, pp. 474-483, 2012.

Baker, P.N., et al., The role of pain and function in determining patient satisfaction after total knee replacement. Data from the National Joint Registry for England and Wales. J Bone Joint Surg Br, 89(7): p. 893-900, 2007.

Bellemans, J., et al., Fluoroscopic analysis of the kinematics of deep flexion in total knee arthroplasty. Influence of posterior condylar offset. J Bone Joint Surg Br, 84(1): p. 50-3, 2002.

Beswick, A.D., et al., What proportion of patients report long-term pain after total hip or knee replacement for osteoarthritis? A systematic review of prospective studies in unselected patients. BMJ Open, 2(1): p. e000435, 2012.

Bloemker, K.H., et al., Computational knee ligament modeling using experimentally determined zeroload lengths, Open Biomedical Engineering Journal, vol. 6, no. 1, pp. 33-41, 2012.

Bourne, R.B., et al., Patient satisfaction after total knee arthroplasty: who is satisfied and who is not? Clin Orthop Relat Res, 468(1): p. 57-63, 2010.

Carr, B.C. and T. Goswami, Knee implants-Review of models and biomechanics. Materials \& Design,30(2): p. 398-413, 2009.

Chen, Z., et al., Prediction of in vivo joint mechanics of an artificial knee implant using rigid multibody dynamics with elastic contacts, Proceedings of the Institution of Mechanical Engineers, Part H: Journal of Engineering in Medicine, 228 (6), pp. 564-575, 2014.

Dossett, H.G., et al., A randomised controlled trial of kinematically and mechanically aligned total knee replacements: Two-year clinical results, Bone and Joint Journal, vol. 96 B, no. 7, pp. 907-913, 2014.

Ewing, J.A., et al., Estimating patient-specific soft-tissue properties in a TKA knee, Journal of Orthopaedic Research, vol. 34, no. 3, pp. 435-443, 2016.

Galbusera, F., et al., Material models and properties in the finite element analysis of knee ligaments: a literature review, Bioengineering and Biotechnology, vol. 17;2:54, 2014. 
Harris, M.D., et al., A Combined Experimental and Computational Approach to Subject-Specific Analysis of Knee Joint Laxity, Journal of Biomechanical Engineering, vol. 138, no. 8, 2016.

Harman, M.K., et al., Prosthesis alignment affects axial rotation motion after total knee replacement: a prospective in vivo study combining computed tomography and fluoroscopic evaluations. BMC Musculoskelet Disord, 13: p. 206, 2012.

Hutter, E.E., et al., Is there a gold standard for TKA tibial component rotational alignment? Clin Orthop Relat Res, 471(5): p. 1646-53, 2013.

Howell, S.M., et al., Kinematic alignment in total knee arthroplasty definition, history, principle, surgical technique and results of an alignment option for TKA, Arthropaedia, 1:44-53, 2014.

Li, G., et al., A validated three-dimensional computational model of a human knee joint, Journal of Biomechanical Engineering, vol. 121, no. 6, pp. 657-662, 1999.

Marra, M.A., et al., A Subject-Specific Musculoskeletal Modeling Framework to Predict in Vivo Mechanics of Total Knee Arthroplasty, Journal of Biomechanical Engineering, 137 (2), art. no. 020904, 2015.

Roth, J.D., et al., The limits of passive motion are variable between and unrelated within normal tibiofemoral joints, Journal of Orthopaedic Research, vol. 33, no. 11, pp. 1594-1602, 2015.

Werner, F.W., et al., The effect of valgus/varus malalignment on load distribution in total knee replacements. J Biomech, 38(2): p. 349-55, 2005.

Wismans, J., et al., A three-dimensional mathematical model of the knee-joint, Journal of Biomechanics, vol. 13, no. 8, pp. 677-679,681-685, 1980.

\section{Disclosures}

One or more authors are employed or consult to 360 Knee Systems, Sydney. 